\title{
Getting to the meat of the matter: It's about more than sparing muscle
}

\author{
Seth B. Krantz, MD

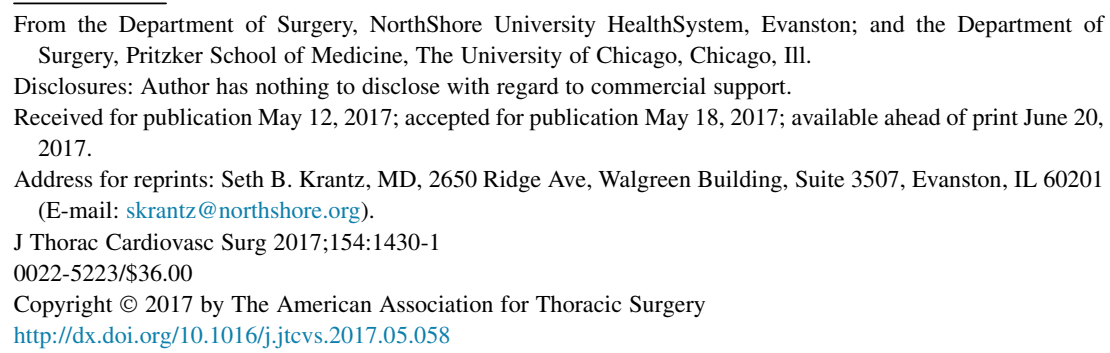

Although the popular debate may be video-assisted thoracoscopic surgery (VATS) versus robotics, many patients with stage I lung disease still undergo thoracotomy. ${ }^{1}$ A frequently made argument is that a "less invasive" thoracotomy, which has rarely been directly compared with VATS, may mitigate the advantage of VATS. To address this, Zhao and colleagues $^{2}$ have performed an excellent, straightforward analysis of 1083 patients with cT1N0M0 disease undergoing lobectomy by either VATS or a muscle-sparing thoracotomy. In their unmatched analysis in this issue of the Journal, VATS was associated with improved short- and long-term outcomes. Thoracotomy, consistent with other reports, ${ }^{3}$ demonstrated better nodal assessment, with double the rate of nodal upstaging. Importantly, the thoracotomy group patients had larger, more central tumors. To address this, Zhao and colleagues ${ }^{2}$ performed a propensity match on 482 patients, in which the VATS group continued to show improved short-term outcomes, with a full day shorter chest tube duration, nearly $700 \mathrm{~mL}$ less chest tube output, a full day shorter length of stay, and a markedly lower complication rate $3.3 \%$ for VATS vs $9.1 \%$ for muscle-sparing thoracotomy). Although patients undergoing thoracotomy still had more nodes assessed, the rate of nodal upstaging was no longer significantly different. Long-term outcomes were also no longer different.

This is a well-done study with some important and notable findings. It adds to the mounting evidence that minimally invasive approaches have better perioperative outcomes for patients ${ }^{4}$ and that this holds true even in direct comparison with a more limited, "less invasive" thoracotomy. It also provides further confirmation that performing a VATS lobectomy does not compromise oncologic outcomes. ${ }^{5}$ Studies done early in the VATS era showed better nodal assessment with higher rates of upstaging in patients undergoing thoracotomy. ${ }^{3}$ This more contemporary series shows that although thoracotomy was still associated with more nodes assessed, upstaging rates were equivalent.

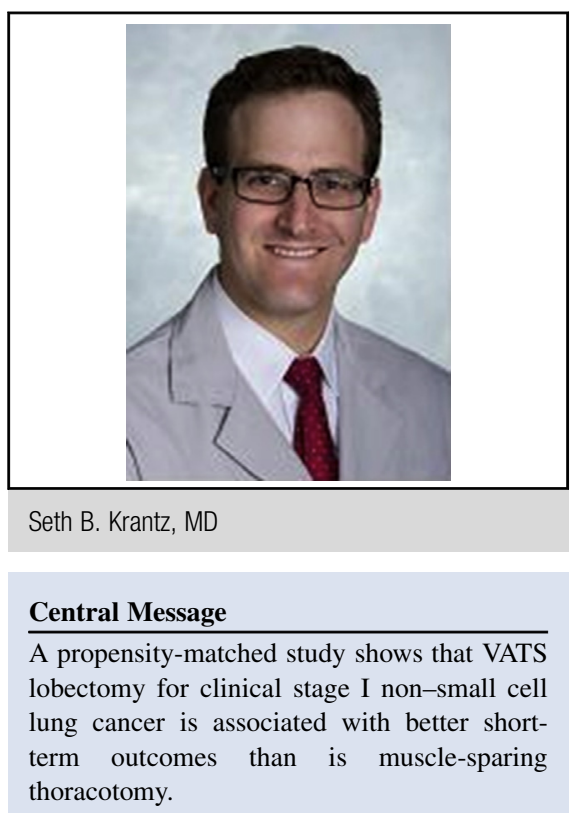

See Article page 1420 .

There are several important limitations. There are significant differences between the groups with respect to tumor size and location. Although Zhao and colleagues ${ }^{2}$ performed propensity matching, this cannot completely adjust for what appears to be a strong underlying selection bias, and how patients were selected for VATS versus thoracotomy is also not clearly elucidated. Second, this was a relatively healthy patient population, with a mean forced expiratory volume in 1 second of nearly $90 \%$. If more patients with limited lung function had been included, the impact of VATS may have been even more significant. Finally, Zhao and colleagues ${ }^{2}$ did not look at prolonged air leak, one of the main drivers of increased length of stay and pulmonary complications, nor did they assess either short- or longterm pain outcomes.

Overall, this is a well-done article with a straightforward question and clear results. Despite the lack of patients with significant chronic obstructive pulmonary disease, Zhao and colleagues ${ }^{2}$ are to be commended on their very low complication rate and even more impressive mortality of $0.09 \%$. With a contemporary series, they show that a thoracoscopic approach, even when compared with a limited thoracotomy, provides significant perioperative benefits to the patients, with excellent 
long-term outcomes, and should be the standard of care for stage I lung cancer.

\section{References}

1. Yang CF, Sun Z, Speicher PJ, Saud SM, Gulack BC, Hartwig MG, et al. Use and outcomes of minimally invasive lobectomy for stage I non-small cell lung cancer in the National Cancer Database. Ann Thorac Surg. 2016;101:1037-42.

2. Zhao Y, Li G, Zhang Y, Hu H, Zhang J, Sun Y, et al. Comparison of outcomes between muscle- sparing thoracotomy and video-assisted thoracic surgery in patients with cT1NOM0 lung cancer. J Thorac Cardiovasc Surg. 2017;154:1420-9.
3. Boffa DJ, Kosinski AS, Paul S, Mitchell JD, Onaitis M. Lymph node evaluation by open or video-assisted approaches in 11,500 anatomic lung cancer resections. Ann Thorac Surg. 2012;94:347-53; discussion 353.

4. Laursen LØ, Petersen RH, Hansen HJ, Jensen TK, Ravn J, Konge L. Video-assisted thoracoscopic surgery lobectomy for lung cancer is associated with lower 30day morbidity compared with lobectomy by thoracotomy. Eur J Cardiothorac Surg. 2016;49:870-5.

5. Berry MF, D'Amico TA, Onaitis MW, Kelsey CR. Thoracoscopic approach to lobectomy for lung cancer does not compromise oncologic efficacy. Ann Thorac Surg. 2014;98:197-202. 